\title{
Identity imposition and its role in a stereokinetic effect
}

\author{
HANS WALLACH and NICHOLAS M. CENTRELLA \\ Swarthmore College, Swarthmore, Pennsylvania
}

\begin{abstract}
Lines of constant curvature, a circle or a straight line, have no distinguishable parts. Yet they are perceived as if they did. When they move and intersect, they are perceived to slide across each other as if one of them had parts that can be seen to move in relation to the other line. With no such parts present in stimulation, they are products of perception. It was found that a third line of constant curvature, the helix, is also seen to slide when two helixes intersect and are in motion. Another manifestation of perceived identical parts is that rotating circles and similar shapes are perceived not to rotate even when cues for rotation are present. Furthermore, changes between merely perceived identical parts can result in apparent depth. Evidence is presented that such depth, known as the stereokinetic effect, results from kinetic depth-effects that are based on perceived identical parts instead of on actually identical parts, and that depth is seen when the intervals between such perceived parts change length and orientation simultaneously.
\end{abstract}

In 1956, Wallach, Weisz, and Adams reported a novel observation, which has been largely forgotten. When a pattern of two homogeneous rings that cross each other (Figure 1) is slowly rotated, the rigid pattern is seen only briefly. Soon, the rings seem to move independently of each other. While they still revolve about the center of the display, each ring ceases to rotate as it did when it was part of the rigid pattern and as it does objectively. When the rings appear to move independently, one ring seems to slide across the other.

If the rings are replaced by different shapes-for instance, a pair of oblongs-nothing like this can happen. As the display turns, its rotation changes the orientation of each oblong and makes its rotation visible. The rings show no such change of orientation, because turning a ring about its center produces no visible change. A circle is a line of constant curvature, and any part of it can be superimposed on any other part; if it is smoothly drawn, a rotation about its center cannot be seen. But neither does it provide cues that it is not rotating; whether a circle turns in itself or not is indeterminate where vision is concerned.

This is only part of the explanation of the strange behavior of the rings. There is a strong tendency to perceive a circle that rotates about its center as not turning. Wallach et al. (1956) reported two impressive demonstrations for this tendency. When a somewhat wider ring with its outer edge smooth and its inner edge scalloped rotates about its center, the outer edge seems to be stationary while the inner edge is seen to move. "This gives rise to the odd impression of a continuous shearing in the sur-

This work was supported by Grant BNS-8318772 from the National Science Foundation to Swarthmore College, Hans Wallach, principal investigator. The authors' mailing address is: Department of Psychology, Swarthmore College, Swarthmore, PA 19081.

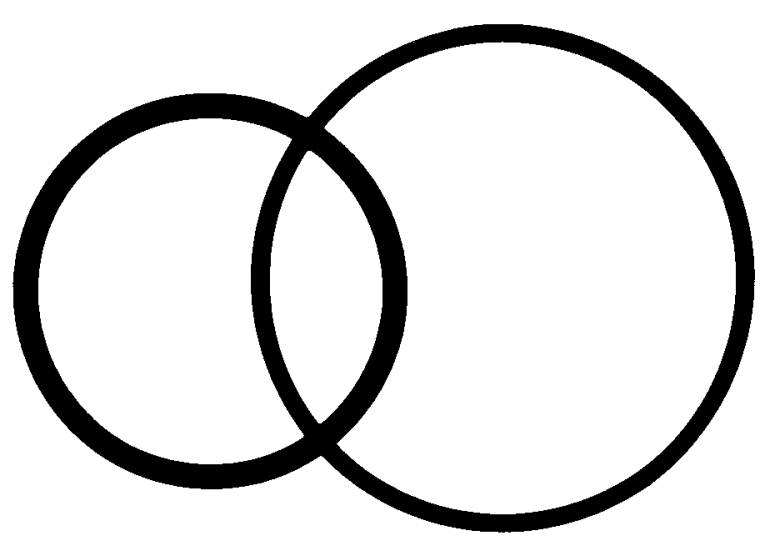

Figure 1. When the pattern of intersecting rings rotates, it is only briefly seen to turn as a whole. Soon the rings seem to move independently of each other.

face of the ring" (Wallach et al., 1956, p. 52). Even when a circular shape has a deformation that makes its changing orientation when it rotates visible, that shape will show the tendency not to rotate. When the shape shown in Figure 2 is made to rotate about the center of the circular section of its outline, it is seen to stand still. Instead, the part of its outline that deviates from the circular shape appears to be a bulge that moves around a circular shape like a wave.

This tendency to perceive a rotating circle as standing still results from the perceived identity of its parts. Inspection of the rotating pattern of two rings (Figure 1) shows this clearly. At the stage where the rings are seen to move independently of each other and one of them seems to slide across the other, paying attention to the crossing point one has the clear impression that one ring moves over the other. "In experience the ring consists of definite parts which move in relation to the other ring'" 


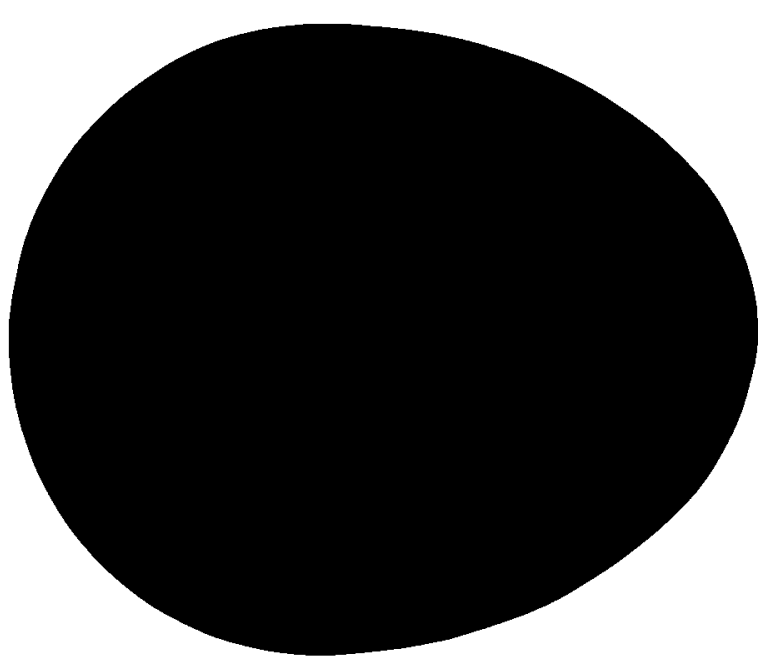

Figure 2. When the egg-shaped figure is rotated about its center, it is readily seen not to turn. The part of the outline that deviates from the perceived circular shape is seen as a bulge that moves around it.

(Wallach et al., 1956, p. 51). Although they are by shape indistinguishable from one another, these parts remain identified. Wallach et al. (1956) called the underlying perceptual function identity imposition.

\section{Other Instances of Identity Imposition}

There are two further lines of constant curvature, a straight line and a helix. Any part of a straight line is congruent with any other part. Under certain circumstances, a straight line also shows the results of identity imposition (Wallach, 1935; for a brief report, see Wallach, 1976, chap. 9, p. 1). Because it is of constant curvature, a smoothly drawn straight line cannot transmit its actual motion direction to the eye when it is laterally displaced in some direction and when its ends are not visible. Nevertheless it is perceived to move in a definite direction-namely, perpendicularly to its extent. ${ }^{1}$ But when part of a moving line is visible in an aperture, a line is seen that ends at the aperture edges. Therefore, when in Figure 3 the oblique line $A$ is in downward motion, its parts that are visible in the upper oblong aperture are perceived to move from right to left, and the same happens when it becomes visible in the lower aperture. When Line B in Figure 3 enters the lower aperture it is perceived to move to the right-that is, in opposite direction to Line A's apparent motion. When the crossing of the lines is visible in the aperture as it is at the moment that Figure 3 depicts, the lines are sill perceived to move horizontally in opposite directions, and when this happens, one line seems to slide across the other, much as the intersecting rings of Figure 1 do. However, the manner in which the identity imposition that this perceived sliding implies comes about is different. In the case of the line, it results from the line in the aperture being perceived as a unitary object, whereas the parts of the rings that are perceived to remain the same are those that lie in the same direction from the rings' centers.

\section{EXPERIMENT 1}

The question arose whether identity imposition operates also in the case of the third line of constant curvature, the helix. Is a section of a helix also perceived as an identical part so that it too appears to slide through a crossing point with another curve when it moves, as intersecting rings do when they revolve?

\section{Method}

Subjects. Fourteen undergraduate students observed.

Equipment and Procedure. A pattern of two intersecting helixes was constructed by winding two strips of black paper tape around a transparent plastic cylinder. The winding of one strip climbed at an angle of $35^{\circ}$ and the other at $20^{\circ}$. The diameter of the cylinder was $10.2 \mathrm{~cm}$, and the cylinder was $42 \mathrm{~cm}$ high. The cylinder was set in a groove of a horizontal plate that was fixed to the vertical slow shaft of a motor. It stood in front of a white wall in a wellilluminated room. It was made to turn clockwise, with a rotation rate of $30 \mathrm{rpm}$.

When the turning cylinder was observed, the windings seemed to move smoothly upward at different speeds. This upward motion is possible because the helical windings are of constant curvature. Where one winding appeared to move in front of another, it seemed to slide. It was our purpose in this experiment to get data on such

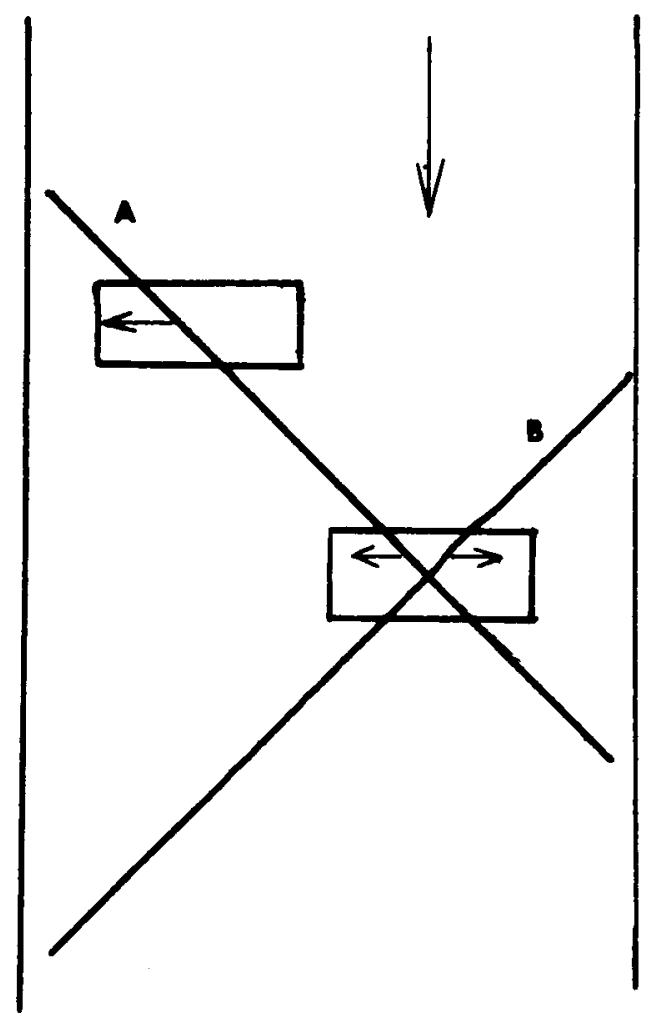

Figure 3. A panel with crossing lines $A$ and $B$ in downward motion visible in two oblong apertures. The horizontal arrows show the perceived motion directions of the lines at the moment depicted. 
sliding motion. To that end, we directed our subjects' attention to a point where the two tapes actually crossed. As the cylinder turns, such a fixed point moves horizontally on a circle around it. Mounted on a separate stand was a thin horizontal rod that ended in front of the cylinder at the level of the circle. The cylinder was turned to the position at which the crossing point was just behind the tip of the rod. The subject, who observed from a distance of $210 \mathrm{~cm}$, was asked to look steadily at the tip of the rod. Then the cylinder was made to turn, and the subject was asked to observe what happened when the intersecting tapes moved by.

\section{Results}

Thirteen of the 14 observers spontaneously reported that one of the tapes appeared to slide over the other. This observation was analogous to the apparent sliding motion in the rotating ring pattern. Thus, all three lines of constant curvature show this manifestation of identity imposition.

\section{BENUSSI'S STEREOKINETIC EFFECT}

In the case of the circle, identity imposition results in a further illusion that has been called stereokinetic effect. Benussi ${ }^{2}$ had discovered that a rotating pattern of two circles, a larger one that rotates about its center and a smaller one inside it that is somewhat displaced (Figure 4), will eventually give rise to perceived depth; the smaller circle will appear to be a good distance in front or in back of the larger circle. Before this depth develops, the circles are often seen in the plane of the display. But as in the case of the two rings, the circles will not appear to rotate: The outer circle will appear to be stationary and the inner circle will revolve inside it, the result of its eccentric location; but it also will not rotate about its center. Identity imposition operates.

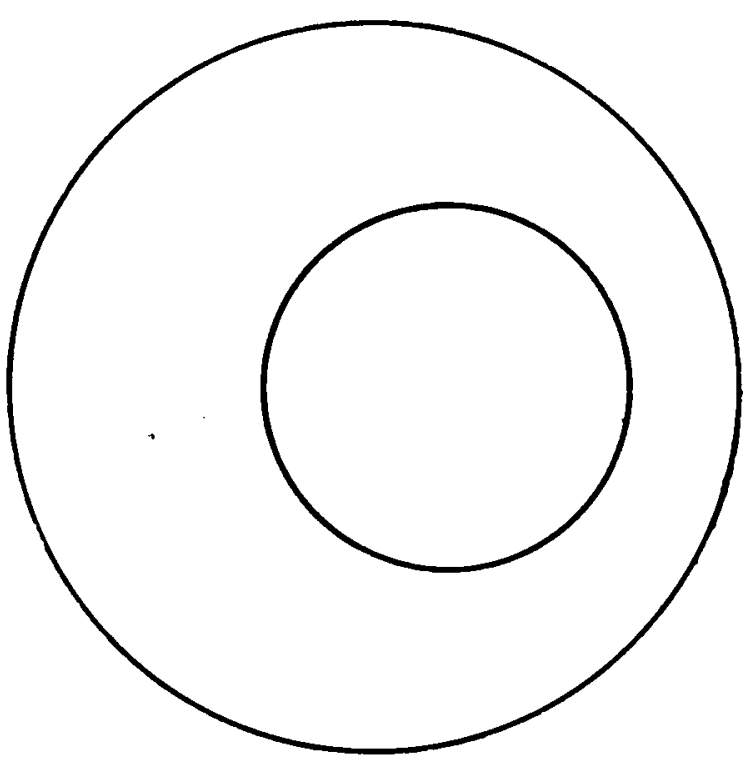

Figure 4. Pattern of two circles showing Benussi's stereokinetic effect when it is rotated.
Wallach et al. (1956) argued that the depth that is subsequently perceived results from a combination of identity imposition and the kinetic depth-effect. But this explanation is hardly ever mentioned in subsequent articles on the Benussi effect, and, if it is, it is not accepted (e.g., Braunstein, 1976, p. 97). The following experiments were intended to clarify this explanation.

The kinetic depth-effect (KDE) operates when, in the absence of other depth cues, a tridimensional shape is perceived when an object undergoes a partial rotation relative to the observer. Ordinarily, this happens when an observer moves so that the direction from which the object is seen changes. In experimentation, the KDE takes effect when the object actually turns. In their investigation of the KDE, Wallach and O'Connell (1953) found the minimal condition under which the KDE takes effect reliably: The retinal image of the turning object must have edges or lines that simultaneously undergo a change in orientation and a change in length. Wallach and O'Connell (1953, Experiment 9) also found that the KDE operates when an array of objects rotates, and when the intervals between the projections of objects on the retina change length and orientation simultaneously. ${ }^{3}$

Under natural conditions that give rise to the $\mathrm{KDE}$, the axes of objects' relative rotation is near the moving observer's frontal plane, but the KDE has also been demonstrated where the rotation axis was in the depth dimension. In Experiment 6 by Wallach and O'Connell (1953), a thin luminous rod was perpendicularly attached to an axle so that its sweep formed the surface of a circular disk when the axle rotated the rod. The rod was positioned in the subject's frontal plane. It was visible in an elliptic aperture in a screen that hid its ends. Therefore, what was seen of the rod when the axle rotated it changed in length as it changed orientation and thus met conditions for a $\mathrm{KDE}$. As a result, a luminous line was perceived rotating in a plane that formed an angle with the frontal plane, with the angle depending on the ratio of the axes of the elliptic aperture.

When in Benussi's display identity imposition causes parts of each circle to be perceived as remaining the same when the circles move in relation to each other, intervals between identical parts on different circles undergo changes in length and orientation simultaneously. (In Figure 5, the lines connecting identical parts of the circles show the changing length and direction of such an interval.) Thus, conditions for a KDE with rotation about the $z$-axis are present. The following experiment demonstrates that a KDE can be obtained under analogous conditions with a shape other than a circle.

\section{EXPERIMENT 2}

\section{Method}

Subjects. Twenty-seven undergraduate students from Swarthmore College were paid for participating.

Apparatus and Displays. In the Benussi display, the smaller circle revolves inside the larger circle but is perceived not to rotate, 

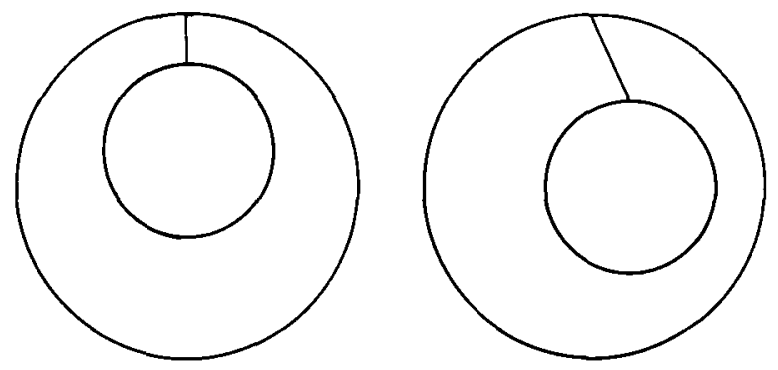

Figure 5. The Benussi circles before and after a clockwise rotation of $90^{\circ}$. The lines that connect points perceived as remaining identical during the rotation change length and direction simultaneously.

although it actually undergoes one rotation during each revolution. Because a shape other than a circle would be seen to rotate as it revolves, the revolving shape-a square in our displays-must be made to rotate once counterclockwise when the shape revolves once clockwise, or vice versa, in order not to rotate. We achieved this by means of a mechanical device, but the display could have been presented on a computer. ${ }^{4}$

Each display consisted of two parts, the outer stationary figure and the inner revolving figure. The outer figure was drawn on a fixed cardboard panel. The inner revolving figure (a circle or a square) was drawn on a smaller cardboard, one circular and the other square, and formed its edge. The center of each of these cardboards could be attached to a shaft that could be made to revolve on a circle with a diameter of $5 \mathrm{~cm}$ and was connected to the driving mechanism in such a way that it did not rotate as it revolved. Hence, when the square cardboard was attached, it retained its upright orientation as it revolved. The panel on which the outer stationary figure was drawn had a circular aperture concentric with the stationary figure. The revolving shaft passed through this aperture, which, with a diameter of $6 \mathrm{~cm}$, had enough room for its circular path. When the inner circular or square cardboard was attached to the shaft, it concealed the aperture. The distance between the panel and the cardboard in the shape of the inner figure was $10 \mathrm{~mm}$. This apparatus was used in four experimental displays that were shown after subjects had seen two displays that introduced them to Benussi's stereokinetic effect. The experimental displays consisted of three KDE displays (Displays 1, 3, and 4) and one stereokinetic display (Display 2), as follows:

Display 1 . The inner and the outer figure were upright squares. The inner one measured $12.8 \mathrm{~cm}$ and the outer one $25.5 \mathrm{~cm}$ on a side. This display was meant to demonstrate a KDE with rotation about the $z$-axis, arranged like the two-circle display of Benussi. But here the intervals that undergo simultaneous changes in length and orientation are between real points, corners of the large and the small square.

Display 2. Both figures were circles. The outer stationary circle had a diameter of $30 \mathrm{~cm}$ and the inner revolving circle one of $16.2 \mathrm{~cm}$. This display was meant to show that our apparatus yielded a depth effect of the same order of magnitude as the analogous Benussi display, for which the subject had given depth estimates earlier.

Display 3. The inner figure was the same circle as in Display 2. The outer circle was replaced by a dot pattern (Figure 6). A demonstration that a depth effect can be obtained when the inner circle revolves in relation to a dot pattern supports our explanation of Benussi's stereokinetic effect-namely, that it results from kinetic depth-effects where intervals between parts of circles perceived as identical change in length and orientation. In the present display, the changing intervals were between such parts of a circle and real dots in the surrounding pattern.
Display 4. The inner figure was the same square as in Display 1. The outer figure was a regular octagon that measured $11.7 \mathrm{~cm}$ on a side. This display differed from Display 1 in that a KDE did not result in a nearly rigid tridimensional shape. Whereas there the two squares form, for every position of the inner one, the end surfaces of a skewed, truncated four-sided pyramid, the combination of square and octagon formed no solid where the edges of the front and the back shape were connected by a surface. Still, each figure has corners that form real points on the two figures. The intervals between these points simultaneously undergo changes in length and orientation.

Introductory displays. Because the Benussi display (Figure 4) does not always yield perceived depth without suggestion, our subjects first observed the four-circle pattern shown in Figure 7 that yields depth almost regularly. The displacement of the smaller circle in

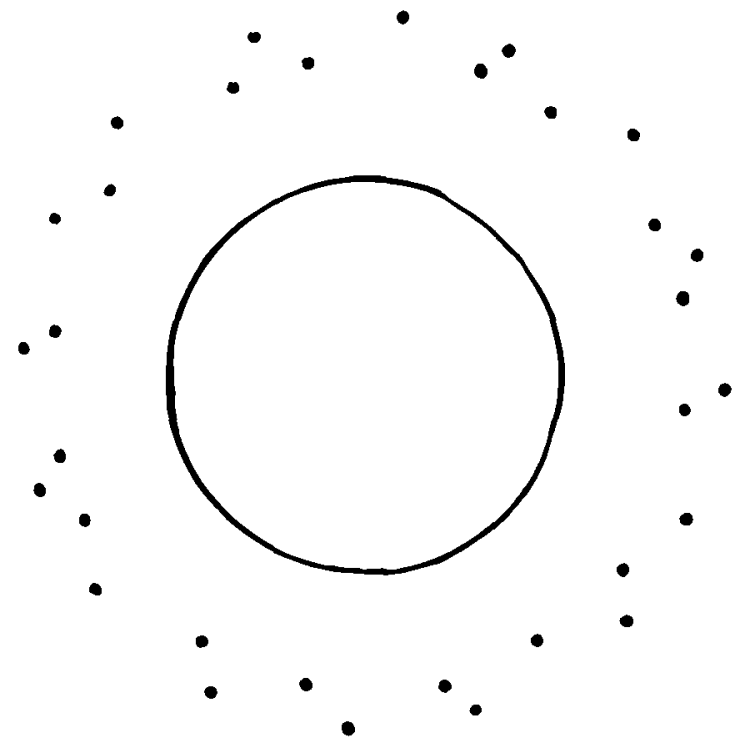

Figure 6. Benussi display with dot pattern replacing a circle.

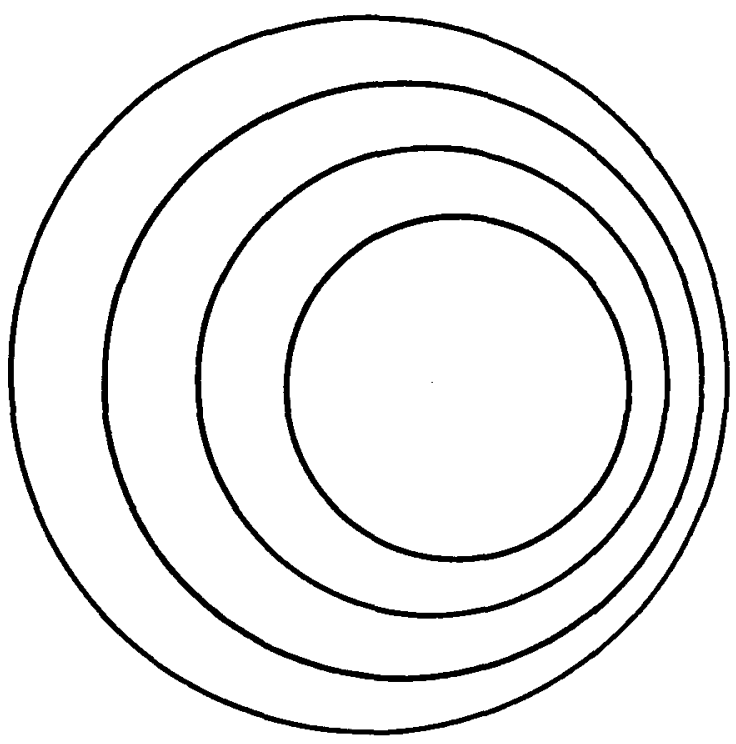

Figure 7. Four circles yielding stereokinetic effect. 
the Benussi display was $2.4 \mathrm{~cm}$, and the largest displacement in the four-circle display, that of the smallest circle, was also $2.4 \mathrm{~cm}$. In the four experimental conditions, the displacement of the inner figures that was provided by the apparatus was $5 \mathrm{~cm}$. In the retinal projections, these displacements were about equal, because in the experimental conditions, the observation distance was twice as large as the distance of the introductory displays $(366$ and $184 \mathrm{~cm})$. The rotation speeds of the introductory displays were $34 \mathrm{rpm}$. They were observed in a well-lit room. The experimental displays required lower and diffuse illumination. When the inner figure cast a shadow on the panel $10 \mathrm{~mm}$ behind it, the shadow provided a cue for this objective depth. We had found that it interfered with the kinetic depth effect that resulted in a larger depth between the inner and outer figure. A lamp that directed the light of a 100 -W bulb mostly upward furnished illumination that did not result in a shadow of the inner figure.

Procedure. All observing in this experiment as well as in the following experiments was monocular. The subject was first shown the four-circle pattern in rotation and was asked to describe what he or she saw. Twenty-six of the 27 subjects almost immediately described a cone of circles. These subjects were then asked to give an estimate of the distance between the near and the far circles by raising a finger of each hand and holding them still until the experimenter had measured how far they were apart. Then subjects were shown the Benussi display (Figure 4), and when they reported seeing depth, they gave estimates in the same manner as before. Here it took longer for some subjects before they reported depth. Observation time was limited to $2 \mathrm{~min}$. Four of the 26 subjects who had seen depth in the four-circle pattern did not report depth in the Benussi display in the allotted time. The 22 subjects who did were then shown the experimental displays.

Eleven subjects observed the four experimental displays in the order (1-4) in which they are listed in Table 1 (Order I). The other 11 subjects made their observations in the opposite order (Order II). Depth estimates were given in the same manner as before and were first obtained after a 10-sec exposure. Regardless of outcome, the subjects gave another estimate after a further exposure of $20 \mathrm{sec}$ and a third estimate after another exposure of $20 \mathrm{sec}$. This procedure provided a rough idea of exposure durations necessary for a $\mathrm{KDE}$ to make its appearance

\section{Results}

Table 1 lists the results for the 22 subjects who reported depth for the introductory Benussi display. In the first part of the table, the numbers of subjects are listed who did not achieve a KDE-that is, those who gave estimates of between $0-$ and $1-\mathrm{cm}$ depth, the latter being the real depth in the displays. No kinetic depth was obtained 14 times out of a total of 66 presentations of kinetic depth displays. The distribution of these no-kinetic-depth cases was not due to strong individual differences. No subject reported three, 5 subjects reported two, and 4 subjects only one case of no kinetic depth.

In the second part of the table, mean depth estimates for the four experimental displays as well as those given by the same 22 subjects for the introductory displays are listed. In computing the former, the highest of the two or three different estimates a subject gave was used..$^{5}$ In the rows labeled Order I and Order II, the mean estimates are listed for the subjects who gave their estimates in those orders. There was no significant order effect.

The last part of the table shows the delays with which the first kinetic depth reports were given in the experimental conditions. It lists the number of subjects who first reported depth of $2 \mathrm{~cm}$ or more during the three exposure periods. More than half of the first depth reports (28 out of 52$)^{6}$ occurred during the first 10 -sec-long exposure, and 16 more during the second period of 20 -sec-long exposures.

The results of the two introductory displays show a striking difference: Mean depth estimates were $43 \%$ higher for the four-circle than for the two-circle display; this difference was significant $(t=3.40, p<.01)$. This happened although the deformation during rotation was the same; the greatest displacement in the four-circle display was the same as the displacement of the smaller circle in the two-circle display. The difference was probably the outcome of conflicting conditions present in every stereokinetic display. The subjects were aware that the circles were drawn on the same surface. The stereokinetic effect was probably stronger when there were four circles, which provided more depth cues. A similar conflict probably diminished the depth estimates that were obtained in the experimental displays; the subjects were aware of the small actual depth between the inner and

Table 1

Results for 22 Subjects Who Were Familiar with Benussi's Stereokinetic Effect

\begin{tabular}{|c|c|c|c|c|c|c|c|}
\hline & \multicolumn{2}{|c|}{ Introductory Displays } & \multicolumn{4}{|c|}{ Experimental Displays } & \multirow[b]{2}{*}{ Total } \\
\hline & Four Circles & Two Circles & Two Squares & Two Circles & Circle/Dots & Square/Octagon & \\
\hline $\begin{array}{l}\text { Number of subjects } \\
\text { did not report depth }\end{array}$ & who & & 4 & 0 & 6 & 4 & 14 \\
\hline $\begin{array}{l}\text { Mean depth estimate } \\
\text { Order I Subjects } \\
\text { Order II Subjects } \\
\text { All Subjects } \\
\text { SD }\end{array}$ & $\begin{array}{r}\text { s (cm) } \\
32.2 \\
33.5 \\
32.9 \\
16.6\end{array}$ & $\begin{array}{l}23.7 \\
22.4 \\
23.0 \\
13.4\end{array}$ & $\begin{array}{l}19.3 \\
23.5 \\
21.4 \\
20.9\end{array}$ & $\begin{array}{l}22.8 \\
28.3 \\
25.5 \\
16.5\end{array}$ & $\begin{array}{l}17.6 \\
18.2 \\
17.9 \\
21.7\end{array}$ & $\begin{array}{l}18.3 \\
16.8 \\
17.5 \\
18.1\end{array}$ & \\
\hline $\begin{array}{l}\text { Number of subjects } \\
\text { first reported kinetic } \\
\text { Period } 1 \\
\text { Period } 2 \\
\text { Period } 3 \\
\end{array}$ & $\begin{array}{l}\text { who } \\
\text { depth }\end{array}$ & & $\begin{array}{r}13 \\
4 \\
1\end{array}$ & $\begin{array}{l}(19) \\
(2) \\
(1)\end{array}$ & $\begin{array}{l}8 \\
8\end{array}$ & $\begin{array}{l}7 \\
4 \\
7\end{array}$ & $\begin{array}{r}28 \\
16 \\
8\end{array}$ \\
\hline
\end{tabular}

Note-Order I and Order II each had 11 subjects. Numbers in parentheses do not contribute to the total reports listed. 
outer figures. Nevertheless, the mean depth estimates show that these displays yielded substantial depth perception.

The mean estimate for the experimental two-circle display (Display 2) was similar to that for the two-circle introductory display. That all subjects reported depth for the experimental display was probably due to the fact that the subjects whose results were listed in the table had been selected for reporting stereokinetic depth for the introductory display. We had apparently succeeded in preventing the gap between the inner and outer circle from interfering with kinetic depth perception.

Experiment Display 1 demonstrated a KDE based on rotation about the $z$-axis. When depth develops in Display 4 , it does not result in a nearly rigid tridimensional arrangement as it does in Displays 1 and 2 . Whereas the two squares or the two circles can be conceived as the end surfaces of a truncated pyramid or a truncated cone, the square and the octagon can be seen only as unconnected surfaces arranged in depth. Nevertheless, the mean depth estimate was only slightly smaller in Display 4 than in Display 1 , with the difference far from significant [ $t(21)$ $=1.39, p<.4]$. Since in Display 4 the changing intervals between the corners of the two figures alone account for the perceived depth, they may well be sufficient to do that also in Display 1. Display 3 supports our explanation of the stereokinetic effect, which assumes that perceived identical parts on different circles participate in a KDE by giving rise to intervals between parts that change simultaneously in length and orientation. In Display 3 , these intervals are between perceived identical parts on the inner circle and real points in the surrounding dot pattern.

\section{EXPERIMENT 3}

In Benussi's stereokinetic effect, identity imposition does not only produce the identical parts and the changing intervals between them that give rise to kinetic deptheffects. It is also responsible for the strong tendency of revolving circles not to rotate. When, in Experiment 2, we presented moving arrangements that were analogues to Benussi's display, we took care that the revolving squares also did not rotate. But if the stereokinetic effect is simply a KDE between perceived identical parts, the nonrotation of the revolving squares in Displays 1 and 4 should not be essential. It may be helpful by preventing rapid displacements of the corner points of the revolv- ing inner square, but in principle it should not be required. Our next experiment showed this to be the case.

\section{Method}

Subjects. Thirty-three undergraduates were paid for participating.

Apparatus. The device used in Experiment 2 was altered so that the inner square underwent one rotation during one revolution, as is ordinarily the case when a figure is made to revolve. Otherwise the experimental display was the same as the two-square display (Display 1) of Experiment 2.

Procedure. As in Experiment 2, two introductory displays preceded this experimental display. They were exactly the same as before. To lessen the effect of the larger and hence more rapid displacements of the corners of the inner square when it also underwent rotation, the rotation speed of the experimental display was lowered. Two speeds were used: For 13 subjects, one rotation took $3 \mathrm{sec}$, and for 20 subjects, it took $6 \mathrm{sec}$. Since very similar results were obtained from these groups, combined results will be reported.

\section{Results}

As the first row of Table 2 shows, about half of the 33 subjects who participated in the experiment reported depth due to the kinetic depth-effect-that is, $2 \mathrm{~cm}$ or more. The mean depth for these 17 subjects was half as large, $11.1 \mathrm{~cm}$, as the depth they reported for the Benussi display, $21.9 \mathrm{~cm}$ (last row of Table 2). The mean depth for all 33 subjects of $5.8 \mathrm{~cm}$ (second row of Table 2) was significantly larger than $1 \mathrm{~cm}$-that is, larger than the real depth in the display; $t$ for the difference between $5.8 \mathrm{~cm}$ and $1 \mathrm{~cm}$ was $3.93(p>.001)$. In spite of the much larger distance changes between corner points of the stationary and of the moving square that resulted from the latter's rotation, 17 subjects saw kinetic depth, and, for all 33 subjects, mean perceived depth was highly significant. We conclude that the perceived nonrotation of revolving circles is not a factor in Benussi's stereokinetic effect.

\section{EXPERIMENT 4}

Display 4 in Experiment 2 demonstrated that rotation around the $z$-axis can result in a KDE where the two figures that are arranged in depth do not also form a solid shape. It remains to be shown that the same can occur in a Benussi effect-namely, that circles can be seen arranged in depth without also forming a solid shape.

For such a demonstration, three circles were arranged so that they were displaced in relation to each other in different directions. The outermost circle (A) that rotated about its center had a diameter of $20 \mathrm{~cm}$. The center of

Table 2

Stereokinetic and Kinetic Depth-Effects

\begin{tabular}{lccc}
\hline & Four Circles & Two Circles & Two Squares \\
\hline Number of subjects who did not report depth & 0 & 5 & 16 \\
For 33 Subjects & 31.7 & 24.2 & 5.8 \\
$S D$ & 20.4 & 19.0 & 6.9 \\
For 17 subjects who saw depth in squares & 32.5 & 21.9 & 11.1 \\
$S D$ & 23.5 & 17.6 & 5.8 \\
\hline
\end{tabular}


Table 3

Depth and No-Rigidity Reports and Mean Depth Estimates with Standard Deviations for 18 Subjects Who Saw Depth

\begin{tabular}{lccc}
\hline & & \multicolumn{2}{c}{ Depth } \\
\cline { 2 - 4 } & No. of Subjects & $M$ & $S D$ \\
\hline 1. Three independent circles with depth between them & 7 & 19.3 & 11.9 \\
2. Rigid pair of circles moving independently of the third & & \\
$\begin{array}{l}\text { circle with depth between them } \\
\text { 3. Same as (2) but no depth between them }\end{array}$ & 6 & 23.0 & 7.6 \\
$\begin{array}{l}\text { 4. Depth between three circles but no spontaneous } \\
\text { reports of independent motion }\end{array}$ & 1 & 20.0 & \\
\hline
\end{tabular}

the larger one of the inner circles (B), which had a diameter of $14.5 \mathrm{~cm}$, was displaced by $2 \mathrm{~cm}$ downward, whereas the center of the smallest circle (C), which had a diameter of $8.5 \mathrm{~cm}$, was $2 \mathrm{~cm}$ to the right of being concentric with Circle B. This arrangement was rotated at a speed of $25 \mathrm{rpm}$.

\section{Method}

Subjects. Twenty-one undergraduates observed this display.

Procedure. The subjects viewed this rotating display individually from a distance of $184 \mathrm{~cm}$, in a room lit by a 75-W bulb. They were asked to describe what they saw. After they gave their descriptions, they were asked how many objects they saw.

\section{Results}

Of the 21 subjects who saw this display, 18 reported depth. This result compares well with 22 out of 27 subjects who reported depth for the two-circle introductory display of Experiment 2. Of the 18 subjects who reported depth, 7 subjects saw both inner circles move independently of each other and of the outer circle and reported depth between the three. Seven subjects saw two circles rigidly connected but moving in relation to the third circle, and 6 of them also saw depth between the rigidly connected circles and the third circle; thus only 1 subject reported no depth for this nonrigid interval. The remaining 4 subjects reported depth for all intervals but did not spontaneously report motion between any two circles. These findings are summarized in Table 3, together with the mean depth estimates. There were, then, 13 subjects out of 18 who saw depth between circles that were also seen to move in relation to each other, and only 5 out of 18 subjects who did not report such a combination. The difference is significant $(z=1.89, p<.03)$.

\section{SUMMARY}

Identity imposition has three manifestations: (1) When lines of constant curvature intersect and move in relation to each other, they are perceived to slide across each other although they have objectively no distinguishable parts that can be seen to shift in relation to the crossed line. When sliding is perceived, the parts that change position with respect to the other line are products of perceptual processes. Straight lines that move in apertures in opposite directions and cross as they do so, patterns of intersecting rings that rotate, and intersecting helixes of differ- ent pitch that turn together are examples. (2) Rotating circles are perceived not to rotate and deformed circles like the egg-shaped form of Figure 2 seem to stand still when they rotate, with the deformation moving around it as a bulge. This failure to rotate is also the product of perceptual processes. (3) The third manifestation of identity imposition is Benussi's stereokinetic effect. ${ }^{7}$ When a larger circle and a smaller circle in eccentric location inside it rotate, they likewise are not seen to rotate. Only the changing location of the smaller circle inside the larger one is perceived as motion. Most observers sooner or later perceive the two circles at different depths. Evidence is presented that such depth results from identity imposition and from a KDE that results from rotation about the $z$ axis. It was shown that rotation about the $z$-axis causes a KDE in the case of a nonconcentric arrangement of an inner and an outer square (Experiment 2, Display 1), and that parts on a circle can participate in a KDE; depth was obtained when the inner figure was a circle and the outer one a ring of dots (Experiment 2, Display 3). It was also shown that a KDE can take place when it does not result in a tridimensional shape; identifiable points on figures that remain independent of each other suffice (Experiment 2, Display 4).

A further experiment showed that nonrotation of the revolving inner figure was not necessary for a $\mathrm{KDE}$ to result from rotation about the $z$-axis; a KDE between two squares could develop when the inner square revolved and rotated. Finally, a special arrangement of three circles could result in a stereokinetic effect where depth was seen between circles that were seen to move independently of each other. The stereokinetic effect can thus take place even when it does not result in a nearly rigid tridimensional shape. Thus, when it is combined with identity imposition, the kinetic depth-effect in its simplest formwhere intervals between parts or points change simultaneously in length and orientation-accounts for Benussi's stereokinetic effect.

\section{REFERENCES}

Braunstein, M. L. (1976). Depth perception through motion. New York: Academic Press.

Musatti, C. L. (1924). Sui fenomeni stereocinetici. Archivo Italiano di Psicologia, 3, 105-120.

STUMPF, P. (1911). Über die Abhängigkeit der visuellen Bewegungsempfindung und ihres negativen Nachbildes von den Reizvorgängen auf der Netzhaut. Zeitschrift für psychologie I, 59, 321-330. 
WALLACH, H. (1935). Über visuell wahrgenommene Bewegungsrichtung. Psychologische Forschung, 20, 325-380.

Wallach, H. (1976). On perception. New York: Quadrangle/The New York Times Book Co.

Wallach, H., \& O'Connell, D. N. (1953). The kinetic depth effect. Joumal of Experimental Psychology, 45, 205-217.

Wallach, H., Weisz, H., \& Adams, P. A. (1956). Circles and derived figures in rotation. American Journal of Psychology, 69, 48-59.

Wilson, J. A., Robinson, J. O., \& Higgins, D. J. (1983). Wobble cones and wobble holes: The stereokinetic effect revisited. Perception, 12, 187-193.

\section{NOTES}

1. P. Stumpf (1911) first wrote about this fact.

2. Benussi's experiment was reported by Musatti (1924).

3 . When the projections of objects only change distances, the array of objects too may appear to rotate in depth, but here the objects may also be seen to move in a plane. Depth is seen reliably only when both distance and orientation change simultaneously.
4. Several years before the following displays were designed, Irwin Rock and James Schubert used quite similar displays presented on a computer. They, too, showed figures other than circles revolving and also had them not rotate as they revolved. Dr. Rock reported these experiments, which have not yet been completed, to the senior author in a letter of May 2, 1986.

5. Estimates not indicating kinetic depth-that is, those between 0 and $1 \mathrm{~cm}$-were included in the listed mean.

6. Twenty-two subjects in three displays (Displays 1, 3, and 4) less 14 no-depth reports.

7. Wilson, Robinson, and Higgins (1983) reported observations about the stereokinetic effect made with displays where kinetic depth was contaminated with cues for perspective depth. Hence their observations need not concern us. Since the authors did not refer to the observations Wallach et al. (1956) reported in connection with identity imposition, their speculations on the stereokinetic effect need not be discussed.

(Manuscript received December 1, 1989; revision accepted for publication July 10, 1990.) 